\title{
ECONOMIA DE ENERGIA NO TRANSIENTE DE AQUECIMENTO INICIAL DE REATOR DE POLIMERIZAÇÃO
}

\author{
D. S. LUXO1 ${ }^{1}$, R. GEDRAITE ${ }^{2}$
}

${ }^{1}$ Bolsista PIVIC FAPEMIG/UFU, discente do curso de Engenharia Química

${ }^{2}$ Professor da Faculdade de Engenharia Química da UFU/MG

E-mail para contato: daphneluxo@hotmail.com

\begin{abstract}
RESUMO - O processo típico de produção de resina acrílica consiste em carregar previamente o reator com solvente orgânico, aquecer até a temperatura de reação e então iniciar a transferência simultânea de monômero e iniciador com vazão controlada. O aquecimento do reator estudado neste trabalho é feito através da circulação de fluido térmico quente $\left(300^{\circ} \mathrm{C}\right)$ e/ou frio $\left(30^{\circ} \mathrm{C}\right)$ na serpentina do reator. O controle da temperatura do reator é feito modulando a vazão do fluido de aquecimento. As temperaturas da massa reacional e do fluido térmico apresentam significativa oscilação durante a etapa inicial de aquecimento, podendo provocar a trinca no material de construção da jaqueta de aquecimento. O conhecimento do comportamento da temperatura da massa reacional é muito importante para permitir o correto controle do processo e o aumento da vida útil da serpentina. $\mathrm{O}$ problema estudado neste trabalho consistiu no modelamento matemático simplificado da temperatura da massa desenvolvido a partir das equações de balanço global de energia aplicado à serpentina e ao reator e foi validado por meio da comparação entre os valores das temperaturas da massa reacional e da serpentina medidos experimentalmente e disponíveis na literatura. Com base nos resultados obtidos, pode-se constatar que o modelo matemático desenvolvido neste trabalho representou de maneira satisfatória o comportamento do processo estudado e permitiu calcular o consumo de energia posta em jogo, visando a sua minimização.
\end{abstract}

\section{INTRODUÇÃO}

O processo tradicional para a produção de resina acrílica consiste em alimentar inicialmente o reator com solvente orgânico, promover o aquecimento do sistema até a temperatura de reação igual a $150{ }^{\circ} \mathrm{C}$ e então iniciar a transferência simultânea de monômero e de iniciador sob vazão controlada. Tipicamente, decorridos alguns minutos após a transferência do monômero e do iniciador tem-se inicio a fase de reação exotérmica.

O fluxograma simplificado apresentado na Figura 1 ilustra este processo, o qual é chamado de "dual feeding" porque o monômero e o iniciador ficam em tanques separados e são transferidos simultaneamente ao reator com vazão controlada. 
As etapas de aquecimento e de resfriamento do reator são realizadas através da circulação de fluido térmico quente $\left(300^{\circ} \mathrm{C}\right)$ e/ou frio $\left(30^{\circ} \mathrm{C}\right)$ na serpentina meia-cana do reator. $\mathrm{O}$ controle da temperatura do reator é feito por meio de malhas de controle das temperaturas da massa reacional e do fluido de aquecimento/resfriamento com base na atuação sobre a vazão de fluido térmico alimentado ao sistema.

No presente cenário de oferta de energia, seja no Brasil ou em qualquer outro país, restrições de ordem financeira e ambiental se conjugam de modo a incrementar os custos das fontes de energia e configuram perspectivas preocupantes de descompasso entre as disponibilidades e as demandas energéticas, ampliando significativamente a importância do uso racional de energia. Trata-se de uma via relativamente pouco explorada e com interessantes possibilidades, pois em muitas plantas industriais o consumo de energia representa uma parcela importante dos custos de produção.

Figura 1 - Fluxograma simplificado do processo em estudo

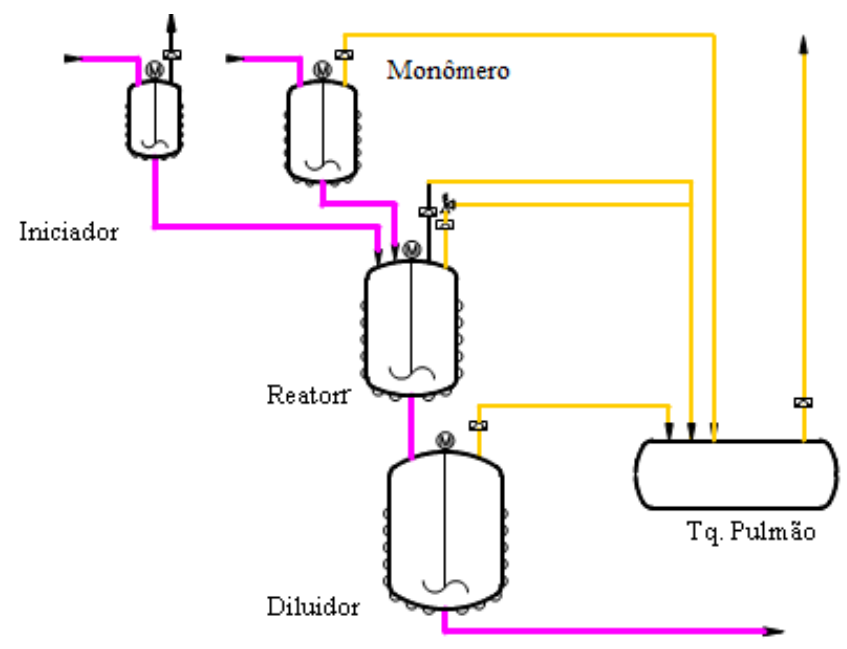

\section{DESCRIÇÃO DO PROBLEMA}

Na reação de polimerização estudada, o solvente alimentado ao reator foi inicialmente aquecido até a temperatura de reação. Este processo de aquecimento, conduzido de modo gradual e contínuo, consiste na elevação da temperatura do fluido de aquecimento empregado, um óleo térmico. Este recebia um aquecimento mais intenso de início, por aproximadamente 500 segundos, após o que tinha a sua temperatura elevada a uma taxa mais lenta, considerando-se uma diferença aproximadamente constante entre a temperatura do óleo térmico e a temperatura do meio reacional de $60^{\circ} \mathrm{C}$. Quando a temperatura da massa no interior do reator estava bem próxima do set point (diferença de aproximadamente $5{ }^{\circ} \mathrm{C}$ ), a temperatura do óleo era bruscamente reduzida para aproximadamente $125^{\circ} \mathrm{C}$ visando minimizar o efeito da oscilação da temperatura do meio reacional em torno do respectivo set point imediatamente antes do inicio da etapa de polimerização. Esta redução da temperatura do óleo térmico era obtida por meio da introdução de óleo térmico a $30{ }^{\circ} \mathrm{C}$. Na Figura 2 é apresentado o comportamento temporal das temperaturas da massa reacional e de entrada e de saída do óleo térmico obtido a partir de dados de operação real do reator (Milan et al, 2013). 
Esta maneira de conduzir a operação do reator apresentava alguns problemas técnicos relacionados com o aparecimento de fissuras nas soldas dos componentes da jaqueta meia cana, devido ao choque térmico provocado pela alteração brusca na temperatura do óleo térmico alimentado, além de implicar na necessidade de descartar quantidade significativa de energia gasta no aquecimento (Milan et al, 2013).

Figura 2 - Comportamento temporal das temperaturas da massa e da camisa do reator em estudo no cenário original de operação

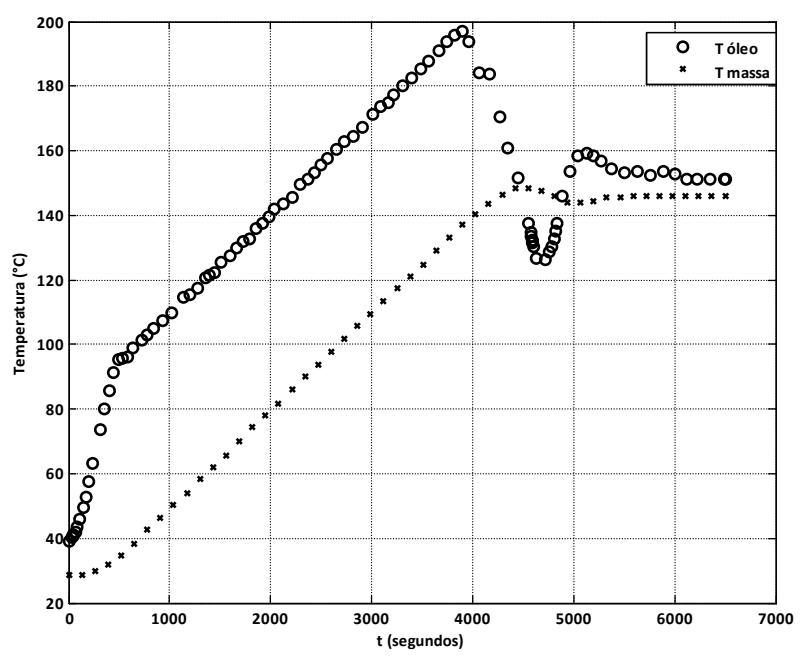

\section{ANÁLISE DO CENÁRIO DE ECONOMIA DE ENERGIA}

Analisando o cenário original de modo a buscar uma alternativa para minimizar o problema do aparecimento de fissuras nas soldas da camisa, foi proposta uma nova maneira de se operar o reator durante a etapa de aquecimento inicial. Foi realizado o cálculo da quantidade de calor disponível na jaqueta, bem como o calculo da quantidade de calor necessária para que a massa atinja o valor desejado, em tempo real (VDI, 2010; Chopey, 2006). Deste modo, foi possível interromper a alimentação de óleo térmico quente (a $300{ }^{\circ} \mathrm{C}$ ) no momento em que a quantidade de calor disponível na jaqueta for a necessária e suficiente para fazer com que a massa reacional atinja a temperatura de reação requerida, dispensando a necessidade de alimentar o óleo térmico frio $\left(\mathrm{a} 30^{\circ} \mathrm{C}\right)$.

Um modelo matemático simplificado foi desenvolvido para estimar a temperatura da massa reacional em cada instante e, a partir deste valor, calcular a quantidade de calor necessária e a disponível, como citado no parágrafo anterior (Bouchenchir et al, 2013; Milan et al, 2013). Deste modo, não mais ocorria o desperdício de calor presente na jaqueta de aquecimento, pois após atingir a temperatura adequada de operação, o modelo coloca o sistema de óleo térmico em recirculação até que este troque todo o calor disponível com a massa reacional. Além do importante cenário de economia de energia, este novo modelo proposto evita o surgimento de fissuras nas soldas da jaqueta, visto que a variação brusca de temperatura não existe mais. Este modelo foi simulado no ambiente Simulink ${ }^{\circledR}$ do aplicativo Matlab ${ }^{\circledR}$. Na Figura 3 é apresentado o diagrama de simulação utilizado neste trabalho e na Figura 4 o comportamento temporal das temperaturas da massa reacional e da camisa do reator em estudo no novo cenário proposto de operação. 
Figura 3 - Diagrama de simulação utilizado no trabalho

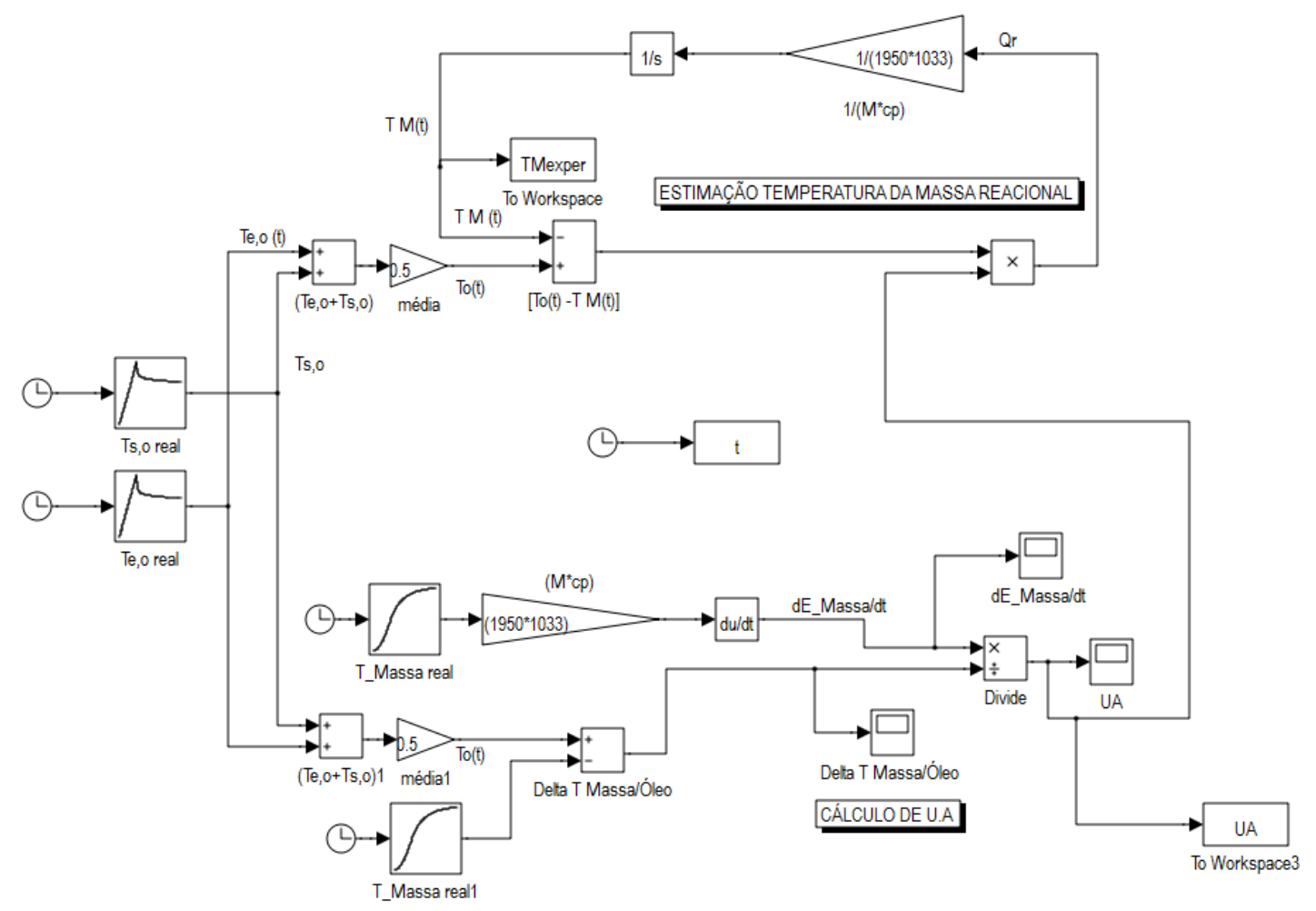

Figura 4 - Comportamento temporal das temperaturas da massa e da camisa do reator em estudo no novo cenário de operação

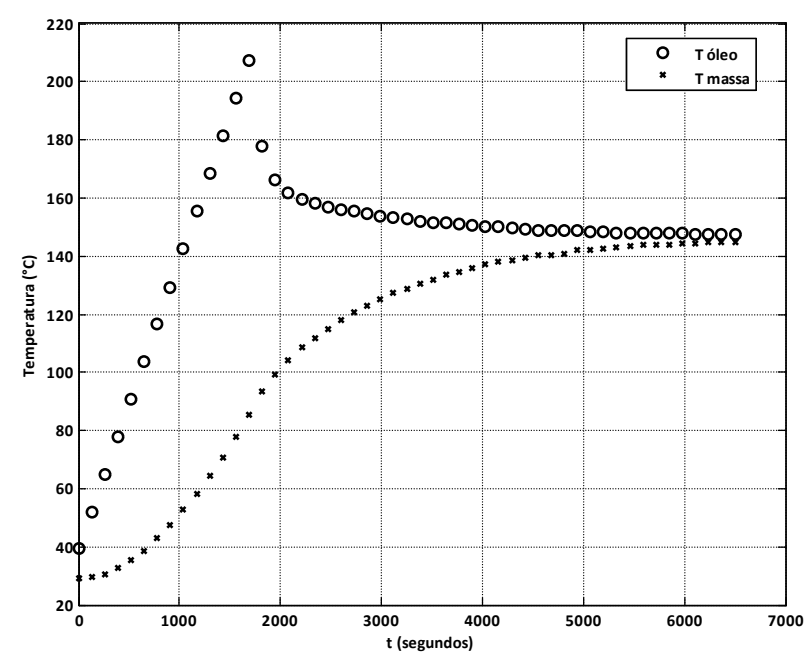

\section{RESULTADOS \& DISCUSSÃO}

$\mathrm{Na}$ Figura 5 são apresentadas as curvas que representam o comportamento temporal da temperatura da massa reacional para os dois cenários considerados neste trabalho. Pode-se perceber que na estratégia de aquecimento original a temperatura da massa no instante $\mathrm{t}=$ 6500 segundos é Tmassa original $=146,1{ }^{\circ} \mathrm{C}$ ao passo que na estratégia de aquecimento 
modificado, a temperatura da massa no instante $t=6500$ segundos é Tmassa modificado $=$ $145^{\circ} \mathrm{C}$. A diferença entre ambas é desprezível, implicando em um erro relativo menor do que $1,0 \%$, o que é totalmente aceitável em um reator de polimerização de escala industrial.

Na operação conduzida no novo cenário proposto de operação, a temperatura da massa varia mais rapidamente em relação ao cenário original, o que tende a ser benéfico para a operação do reator, uma vez que favorece a economia de energia. Além do mais, no cenário original de operação ocorre um pequeno sobressinal em relação à temperatura final de operação, de valor igual a $148,5^{\circ} \mathrm{C}$, o que deve ser evitado por favorecer a ocorrência do indesejado choque térmico na camisa do reator.

O consumo médio de energia no sistema foi avaliado considerando-se dois cenários, a saber: cenário 1 no qual se usou a estratégia original de operação e cenário 2 no qual se usou a estratégia modificada de operação (Gedraite et al, 2011). Na Tabela 1 são apresentados os resultados obtidos neste trabalho.

Figura 5 - Comportamento temporal das temperaturas da massa em ambos os cenários de operação

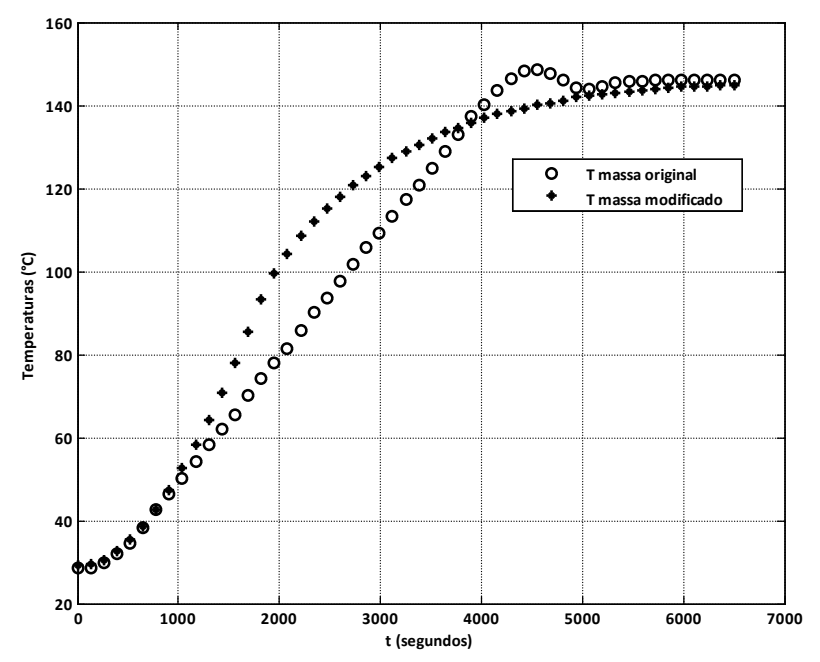

Tabela 1 - Cálculo comparativo de consumo de energia na fase de aquecimento

\begin{tabular}{cccccccc}
\hline Ensaio & Cenário & $\begin{array}{c}\Delta \mathrm{t} \\
(\mathrm{s})\end{array}$ & $\begin{array}{c}\text { Tmassa } \\
\text { Inicial } \\
\left({ }^{\circ} \mathrm{C}\right)\end{array}$ & $\begin{array}{c}\text { Tmassa } \\
\text { Final } \\
\left({ }^{\circ} \mathrm{C}\right)\end{array}$ & $\begin{array}{c}\Delta \mathrm{T} \text { massa } \\
\left({ }^{\circ} \mathrm{C}\right)\end{array}$ & $\begin{array}{c}\text { Energia } \\
\text { gasta } \\
(\mathrm{J})\end{array}$ & $\begin{array}{c}\text { Energia } \\
\text { gasta por }{ }^{\circ} \mathrm{C} \\
\left(\mathrm{KWh} /{ }^{\circ} \mathrm{C}\right)\end{array}$ \\
\hline \hline 1 & Original & 4568 & 30,4 & 150,4 & 120 & $9,63 \mathrm{E}+08$ & 2,23 \\
2 & Original & 4028 & 36 & 147,5 & 111,5 & $7,65 \mathrm{E}+08$ & 1,91 \\
3 & Original & 4100 & 33 & 147,9 & 114,9 & $8,07 \mathrm{E}+08$ & 1,95 \\
4 & Original & 3820 & 39 & 148,2 & 109,2 & $7,96 \mathrm{E}+08$ & 2,02 \\
5 & Modificado & 2559 & 62,3 & 139 & 76,7 & $4,03 \mathrm{E}+08$ & 1,46 \\
6 & Modificado & 3900 & 69,83 & 144,3 & 74,47 & $4,19 \mathrm{E}+08$ & 1,56 \\
7 & Modificado & 3582 & 58,23 & 147,1 & 88,87 & $4,66 \mathrm{E}+08$ & 1,46 \\
8 & Modificado & 3400 & 32,2 & 146,9 & 114,7 & $5,63 \mathrm{E}+08$ & 1,36 \\
\hline
\end{tabular}


Com base nos resultados apresentados na Tabela 1, o processo de aquecimento conduzido com base na estratégia modificada de operação apresentou um consumo médio de energia por unidade de variação de temperatura de $1,46 \mathrm{kWh} /{ }^{\circ} \mathrm{C}$. Já no processo conduzido na estratégia original de operação, o consumo médio foi de $2,02 \mathrm{kWh} /{ }^{\circ} \mathrm{C}$. Portanto, foi obtida uma economia de aproximadamente $28 \%$ no consumo de energia na etapa inicial de aquecimento do reator de polimerização.

\section{CONCLUSÃO}

Como resultado da utilização do modelo proposto por Milan et al (2013), obteve-se significativa economia de energia na fase de aquecimento da massa reacional até que a temperatura de reação fosse alcançada. O modelo desenvolvido é bastante simples e de fácil implementação em sistemas de controle industriais. A economia de energia proporcionada pelo emprego desta metodologia sugere um caminho promissor para o aumento da eficiência energética dos processos da indústria química.

\section{AGRADECIMENTOS}

Os autores agradecem à UFU e à FAPEMIG (Fundação de Amparo à Pesquisa do Estado de Minas Gerais) pelos recursos concedidos e apoio em pesquisas no projeto $\mathrm{N}^{\mathrm{o}}$ ICFAPEMIG2014-0090.

\section{REFERÊNCIAS}

BOUCHENCHIR H., CABASSUD M., LE LANN M.V., CASAMATTA G. (2001) A General Simulation Model and a Heating/Cooling Strategy to Improve Controllability of Batch Reactors, In: Trans IChemE, Vol.79, Part A, France.

CHOPEY, N. P. (2006) Handbook of Chemical Engineering Calculation, 3rd Edition, McGraw-Hill, USA.

GEDRAITE R., MILAN W., KUNIGK. L. e GEDRAITE, E. S. Modelo matemático usado como sensor inferencial para avaliação de economia de energia em reator de polimerização semi-batelada. In: Anais do IV Congresso Brasileiro de Eficiência Energética. Juiz de Fora - MG, 2011.

MILAN, W. GARCIA, C., FERREIRA, N. L., KUNIGK, L., LOBATO, F. S., NEIRO, S. M. S., QUEIROZ, C. G. e GEDRAITE, R. Uso de sensor virtual para controle de transiente de temperatura em reator de polimerização semi-batelada. In: Petro \& Química - Ano XXXV - no 348, São Paulo - SP, 2013.

VDI GESELLSCHAFT (GVC),VDI- Heat Atlas, Berlin, Springer-Verlag, $2^{\text {th }}$ edition, 2010. 